\title{
Efficacy and safety of combined ethinyl estradiol/ drospirenone oral contraceptives in the treatment of acne
}

This article was published in the following Dove Press journal:

International Journal of Women's Health

27 November 2009

Number of times this article has been viewed

Jerry KL Tan'

Chemanthi Ediriweera ${ }^{2}$

'University of Western Ontario and Windsor Clinical Research Inc., Windsor, Ontario, Canada; ${ }^{2}$ University of Western Ontario, Southwest

Ontario Medical Education Network, Windsor, Ontario, Canada

Correspondence: Jerry Tan

2224 Walker Road, Suite 300,Windsor,

Ontario, Canada N8W 5 L7

$\mathrm{Tel}+|51997| 7693$

Fax + I 5199717594

Email jerrytan@bellnet.ca

\begin{abstract}
Acne is a common disorder affecting the majority of adolescents and often extends into adulthood. The central pathophysiological feature of acne is increased androgenic stimulation and/or end-organ sensitivity of pilosebaceous units leading to sebum hypersecretion and infundibular hyperkeratinization. These events lead to Propionibacterium acnes proliferation and subsequent inflammation. Hormonal therapy, including combined oral contraceptives (OCs), can attenuate the proximate androgenic trigger of this sequence. For many women, hormonal therapy is a rational option for acne treatment as it may be useful across the spectrum of severity. Drospirenone (DRSP) is a unique progestin structurally related to spironolactone with progestogenic, antimineralocorticoid, and antiandrogenic properties. It is available in 2 combined OC preparations (30 $\mu \mathrm{g}$ EE/3 mg DRSP; Yasmin ${ }^{\circledR}$ in a 21/7 regimen; and $20 \mu \mathrm{g}$ EE/3 mg DRSP; $Y^{\circledR} z^{\circledR}$ in a 24/4 regimen). These preparations are bereft of the fluid retentional side effects typical of other progestins and their safety has been demonstrated in large epidemiological studies in which no increased risk of vascular thromboembolic disease or arrhythmias was observed. In acne, the efficacy of DRSP-containing OCs has been shown in placebo-controlled superiority trials and in active-comparator non-inferiority trials.
\end{abstract}

Keywords: acne vulgaris, combined oral contraceptives, drosperinone, ethinyl estradiol, efficacy, safety, treatment

\section{Introduction}

Acne vulgaris is a common condition affecting up to $93 \%$ of adolescents and teens $\mathrm{s}^{1,2}$ and can persist into adulthood in $53 \%$ of women and $40 \%$ of men. ${ }^{3,4}$ Women are more likely to report having acne and to have it persist into later years with a mean duration in affected adult women of 20 years. ${ }^{5}$

Due to involvement of visible anatomical sites such as the face and upper torso, along with the potential for chronicity, acne has been associated with a wide spectrum of psychological and social dysfunction including depression, anxiety, suicidal ideation, somatization, embarrassment, and social inhibition. ${ }^{6-8}$ In particular, adult women have been shown to be adversely impacted by the effect of acne on their quality of life. ${ }^{9}$

Hormonal therapies, including those with and without oral contraceptive (OC) properties, have been used by dermatologists as a treatment for acne vulgaris for over 4 decades. For many women, the use of hormonal therapy is a rational option for acne treatment as they may be used across the spectrum of severity. OCs may be used for mild acne in women desiring a form of contraception, and in moderate acne as a primary form of therapy. In severe acne, they are frequently used as one of 2 prerequisite forms of contraception for those initiating oral isotretinoin. ${ }^{10}$ 
The non-contraceptive hormonal options include spironolactone, cyproterone and flutamide. ${ }^{11}$ Of these, spironolactone - a synthetic steroid which binds to androgen receptors, inhibits 5 alpha-reductase activity, and reduces androgen biosynthesis - has been used for over 3 decades in the treatment of acne and has a long record of safety. ${ }^{12}$ Prior to the current era of evidence-based medicine, experiential support suggested the efficacy and relative safety of these agents. Since then, OCs have evolved to contain less estrogen, thereby enhancing their safety and tolerability profile, and to include progestins with low or antiandrogenic potential to improve efficacy in acne. Furthermore, the past decade has witnessed an increase in quality of evidence to support the use of these agents in treatment of acne. Based on clinical trial evidence, the United States Food and Drug Administration (FDA) has approved the following combined OC preparations for treatment of moderate acne: ethinyl estradiol (EE) 20/30/35 $\mu \mathrm{g}$ plus norethindrone $1 \mathrm{mg}$ (Estrostep $\mathrm{Fe}^{\circledR}$; Warner-Chilcott), EE $35 \mu \mathrm{g}$ plus norgestimate 180/215/250 $\mu \mathrm{g}$ (Ortho Tri-Cyclen ${ }^{\circledR}$; Janssen-Ortho), and EE $20 \mu \mathrm{g}$ plus drospirenone $3 \mathrm{mg}\left(\right.$ Yaz $^{\circledR}$; Bayer). In Canada, the corresponding list approved by Health Canada also includes EE $35 \mu \mathrm{g}$ /cyproterone acetate $2 \mathrm{mg}$ (Diane-35 ${ }^{\circledR}$; Bayer; not officially indicated in Canada as an OC), EE $20 \mu \mathrm{g} /$ levonorgestrel $100 \mu \mathrm{g}$ (Alesse ${ }^{\circledR}$; Wyeth Pharmaceuticals), and EE $35 \mu \mathrm{g} /$ drospirenone $3 \mathrm{mg}$ (Yasmin ${ }^{\circledR}$; Bayer). The most recently approved of these are OCs in which the progestin component is drosperinone (DRSP), a derivative of spironolactone. This review focuses on the evidence supporting the use of these agents in the treatment of acne.

\section{Acne pathogenesis and hormonal treatment}

The pathogenesis of acne is conceptualized to involve androgenstimulated hyperkeratinization of the follicular infundibulum and sebaceous hypersecretion; Propionibacterium acnes proliferation within the anaerobic milieu of the pilosebaceous duct, and subsequent inflammation. Effective treatments would ideally target multiple pathogenic factors simultaneously and/or abrogate the initiating event such as androgenic stimulation of pilosebaceous structures. ${ }^{13}$ While each of these modalities targets specific distal pathogenic factor(s), only hormonal therapy ameliorates proximate androgenic stimulation.

\section{Mechanism of action of combined OCs in acne}

Combined OCs are comprised of estrogenic and progestogenic components. Their antiandrogenic potential in acne results from a variety of mechanisms (see Figure 1). Suppression of pituitary gonadotrophin secretion reduces androgen production by the ovaries. Additionally, combined OCs directly inhibit androgenesis by the ovaries and adrenal glands. ${ }^{14}$ The estrogenic component of these agents increase hepatic production of sex hormone binding globulin (SHBG) which binds to testosterone, thereby reducing levels of bioavailable testosterone. The progestin component inhibits 5- $\alpha$ reductase activity, reducing the conversion of testosterone at end-organs to the more biologically potent dihydrotestosterone (DHT). Furthermore, some progestins act as inhibitors to DHT and other androgens by competitive binding to androgen receptors. ${ }^{14}$ Recent studies have shown that androgen receptors can be found at the basal layer of sebaceous glands of the skin and that skin and sebaceous glands contain the biochemical substrates for androgen production and metabolism. Major isozymes of steroid metabolizing enzymes can be found in human sebaceous glands and androgens can be produced within them from adrenal-derived dehydroepiandrosterone sulfate. ${ }^{15}$ Accordingly, the antiandrogenic effects of some progestins may also be mediated locally by inhibiting autocrine stimulation of sebaceous glands by androgens.

\section{Pharmacology of drosperinone}

Drosperinone $(6 \beta, 7 \beta, 15 \beta, 16 \beta$-dimethylene-3-oxo $17 \alpha$ pregn-4-ene-21,17 carbolactone), an analog of spironolactone, is a synthetic progestin uniquely combining progestogenic activity with antimineralocorticoid and antiandrogenic properties. ${ }^{16-18}$ It is the only progestin currently available that it is neither derived from $17 \alpha$-acetoxyprogesterone nor 19-nortestosterone and is thus devoid of androgenic effects of the latter. While comprehensive reviews of the pharmacology of DRSP are available elsewhere, ${ }^{19-21}$ this section focuses on features salient to clinicians.

The progestogenic potency of DRSP has been studied in several animal models where it was found to be within the potency range of cyproterone acetate and norethisterone acetate. ${ }^{16}$ In humans, DRSP been shown to have strong central and peripheral progestational activity ${ }^{17}$ rendering it suitable for oral contraception and hormonal replacement therapy. Additionally, no drug interactions were noted between DRSP and EE that would interfere with the progestogenic or antimineralocorticoid activity of DRSP. ${ }^{16}$

In healthy women, estrogen activates the renin-angiotensin system with attendant effects on sodium and fluid retention. In contrast, progesterone antagonizes some of these aldosterone-mediated effects by high affinity binding 


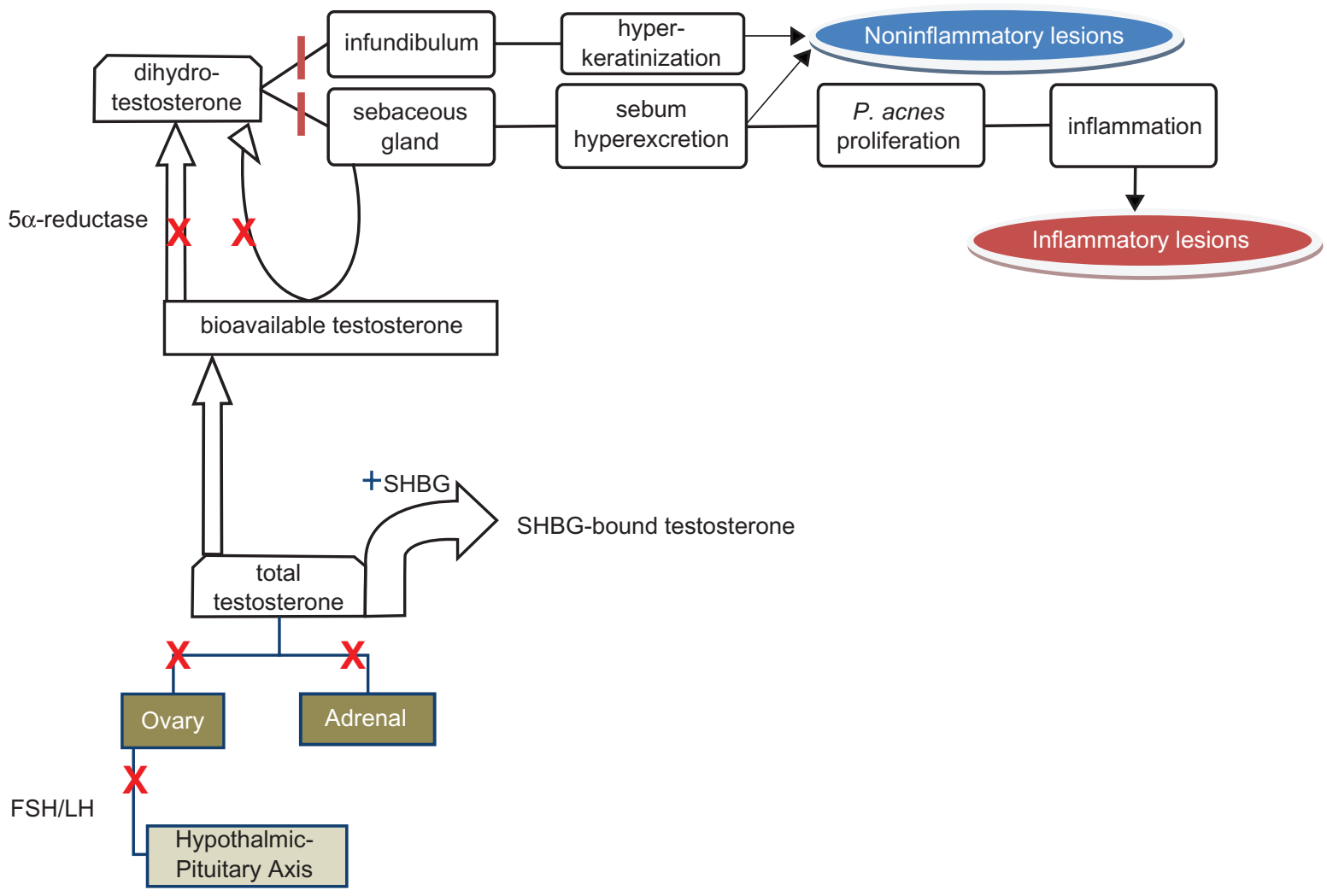

Figure I Androgens in acne pathogenesis and the countervailing effects of combined oral contraceptives (OCs) and antiandrogens. Gonadotropin stimulation of ovaries leads to increased androgenesis. Testosterone is derived from ovarian and adrenal glands. Sex hormone binding globulin (SHBG) binds to testosterone thereby reducing the fraction available for biological activity. Conversion of testosterone to dihydrotestosterone (DHT), the more potent tissue-active metabolite, is mediated by $5 \alpha$ reductase in tissues. DHT increases sebum excretion from sebaceous glands and induces epidermal hyperkeratinization at the infundibulum of pilosebaceous units. These events lead to the formation of comedones. Subsequent proliferation of $P$. acnes bacteria leads to inflammation, manifested as inflammatory lesions of acne. Combined OCs act to block certain portions of the androgenic pathway $(\mathbf{X})$ and enhance SHBG production $(+)$. Antiandrogens, including DRSP, induce androgen receptor blockade at relevant tissue sites (I).

to the mineralocorticoid receptor. DRSP shares the antimineralocorticoid properties of progesterone and its parent compound spironolactone. Compared to spironolactone, the antimineralocorticoid potency of DRSP is approximately eight-fold higher. ${ }^{22}$ Thus, the antimineralocorticoid effect of $3 \mathrm{mg}$ DRSP is equipotent to $25 \mathrm{mg}$ of spironolactone. The absence of antimineralocorticoid activity with other progestins may contribute to fluid retention with associated features of weight gain, breast tenderness, nausea, headache and hypertension..$^{21}$ Therefore, DRSP may ameliorate certain estrogen-induced side effects in women taking OCs. Their use, however, is attended by warnings regarding patients at risk of hyperkalemia including renal insufficiency, hepatic dysfunction, adrenal insufficiency, and in those taking medications affecting potassium levels and the renin-angiotensin system (ACE inhibitors, angiotensin II receptor antagonists, K-sparing diuretics, K supplementation, aldosterone antagonists, and non-steroidal anti-inflammatory agents). ${ }^{23}$

DRSP is antiandrogenic, inhibiting androgen receptormediated transcription in a dose dependent manner. ${ }^{19}$ In vivo experiments of antiandrogenic potency by inhibition of testosterone-induced prostatic growth in juvenile castrated male rats have shown that DRSP has a potency almost ten times greater than progesterone, ${ }^{19}$ one-third that of cyproterone; but superior to that of spironolactone. ${ }^{16,21}$ The antiandrogenic dose equivalency for DRSP compared to spironolactone is undetermined.

The oral bioavailability of DRSP is $\sim 76 \%$ and peak plasma levels are attained in 1-2 hrs. Serum half-life is DRSP is $\sim 31$ hours, while that of EE is 24 hours. Approximately $45 \%$ of an oral dose of DRSP is excreted in the urine and $20 \%$ in feces. While it is not bound to SHBG, up to $97 \%$ is loosely bound to serum albumin. ${ }^{24}$ 


\section{Clinical trials of EE/DRSP OCs in acne}

While more than 25 outcome measures have been used in acne studies, ${ }^{25}$ most have not been evaluated for accuracy or reproducibility. Since the 1980s, however, the Center of Drug Evaluation and Research (CDER) of the FDA approval of new acne treatments has required demonstration of statistically significant superiority in two of three acne lesion count measures (non-inflammatory, inflammatory or total counts) and in static investigator global assessments (IGA). ${ }^{26}$ This approach is based on the clinical relevance of global assessments given the pleomorphic presentation of acne, with varying types of primary lesions in extent and distribution; combined with the greater objectivity and morphologic specificity of lesion counting. Primary lesions in acne are divided into non-inflammatory (comedonal) and inflammatory (papules, pustules, nodules) lesions, while total counts are a summation of these two types. Secondary lesions, which are excluded from primary lesion counting and IGA, comprise post-inflammatory erythema, dyspigmentation and scarring. ${ }^{27}$

At present, DRSP is only available in 2 OC preparations combined with EE $\left(30 \mu \mathrm{g}\right.$ EE/3 mg DRSP; Yasmin ${ }^{\circledR}$ in a conventional 21-day hormone/7-day inert regimen; and $20 \mu \mathrm{g} \mathrm{EE} / 3 \mathrm{mg}$ DRSP; $\mathrm{Yaz}^{\circledR}$ in a 24/4 regimen). The 24 day hormone and 4 day hormone-free interval regimen was developed for $20 \mu \mathrm{g}$ EE/3 mg DRSP in recognition of the greater contraceptive margin of safety with a shorter hormone free interval for low-dose combined OCs. ${ }^{28}$ In this section, we evaluate the evidence supporting the use of these agents in treatment of acne based on placebo-controlled and active-comparator clinical trials.

\section{Placebo-controlled trials}

Two placebo-controlled randomized trials of identical design have been performed for $20 \mu \mathrm{g} \mathrm{EE} / 3 \mathrm{mg}$ DRSP (20EE/DRSP) over 6 cycles. ${ }^{29,30}$ While 2 other publications are cited in the literature, ${ }^{31,32}$ they are both based on the same datasets and analyses as those reported in Maloney et $\mathrm{al}^{29}$ and Koltun et $\mathrm{al}^{30}$ respectively. Accordingly, they will not be discussed further herein. No placebo-controlled trials for $30 \mu \mathrm{g}$ EE/3 mg DRSP (30EE/DRSP) have been published for this indication.

Inclusion criteria in both studies were healthy females aged 14 to 45 years; at least moderate facial acne severity; minimum of 20 inflammatory papules and/or pustules and 20 noninflammatory lesions. Of note is that these acne-specific criteria requested by the FDA were amendments that had originally had greater range of lesion numbers and a severity spectrum that extended to also include mild acne. As these amendments occurred after recruitment, the total number of patients in both trials was greater than that undergoing final efficacy analysis. The IGA was a static scale, not referenced to baseline severity, and based on 6 categories ranging from 0 (normal clear skin) to 5 (many inflammatory and noninflammatory acne lesions). Successful IGA outcomes were the attainment of clear (0) and almost clear (1) severity categories. Exclusion criteria included typical contraindications for combined oral contraceptive use and other acne treatments.

For each study, a sample size of 250 per group was calculated to provide a power of $90 \%$ to detect group differences. Based on the amended criteria, however, the population available for analysis were 218 in the 20EE/DRSP group and 213 in the placebo group in one study ${ }^{29}$ and 228 and 230 , respectively, in the other. ${ }^{30}$ Thus, each of these studies did not achieve the recruitment target based on the power calculation.

Baseline demographics for subjects in both groups in each study were similar. Significant improvements for the 20EE/DRSP groups at end of study were demonstrated for all efficacy outcome measures (see Table 1). Weighted data pooling (see Table 2) for number of subjects from these trials demonstrated the following significant improvements in the active treatment group: $49 \%$ for inflammatory lesions (placebo 33\%); 40\% for noninflammatory lesions (placebo $22 \%$ ), and $44 \%$ for total lesions (placebo $28 \%$ ), $18 \%$ achieving IGA success of clear/almost clear (placebo 6\%).

Adverse events in both trials were characteristic of lowdose combined oral contraceptive therapy and largely mild to moderate in intensity. A greater proportion of subjects in the active arm reported metrorrhagia, menorrhagia, and breast pain. In the active treatment arm of both studies, 2 serious adverse events (depression, pneumonia) were reported but were not considered attributable to study medication. A total of 8 pregnancies in the active treatment arms were reported. Details on 4 were available -2 pregnancies were prior to study medication intake, and 1 was due to non-compliance. ${ }^{29}$ No significant differences in blood pressure or serum potassium levels were observed in either trial between active and placebo groups.

These 2 studies provide the highest level evidence for the efficacy of 20EE/DRSP in treatment of at least moderate facial acne over 6 cycles. Furthermore, the significant improvements in lesion counts and achievement of global success in acne grading were clinically relevant. Shortcomings of these 
Table I Efficacy results of $20 \mu \mathrm{g} E / 3 \mathrm{mg}$ DRSP vs placebo trials in acne

\begin{tabular}{|c|c|c|c|c|}
\hline \multirow[t]{2}{*}{ Treatment arm } & \multicolumn{2}{|c|}{ Maloney et $\mathrm{al}^{29}$} & \multicolumn{2}{|c|}{ Koltun et $\mathrm{al}^{30}$} \\
\hline & Active & Placebo & Active & Placebo \\
\hline $\mathrm{N}$ & 218 & 213 & 228 & 230 \\
\hline \multicolumn{5}{|l|}{ OUTCOMES* } \\
\hline IGA success & $21 \%$ & $9 \%$ & $15 \%$ & $4 \%$ \\
\hline (clear or almost clear) & $(46)$ & $(19)$ & $(35)$ & $(10)$ \\
\hline$\%$ reduction total lesions & $46 \%$ & $31 \%$ & $42 \%$ & $25 \%$ \\
\hline (baseline absolute mean) & $(76)$ & $(76)$ & $(80)$ & $(80)$ \\
\hline$\%$ reduction inflammatory lesions & $51 \%$ & $34 \%$ & $48 \%$ & $32 \%$ \\
\hline (baseline absolute mean) & $(32)$ & $(32)$ & $(33)$ & $(33)$ \\
\hline$\%$ reduction noninflammatory lesions & $42 \%$ & $26 \%$ & $39 \%$ & $18 \%$ \\
\hline (baseline absolute mean) & (44) & (44) & (47) & $(47)$ \\
\hline
\end{tabular}

$* P$ values for all outcomes $<0.001$ versus placebo.

studies include inadequate stratification baseline severity based on IGA and inadequate data on pregnancies in 1 study. ${ }^{30}$

\section{Active-comparator trials}

Two active-comparator trials in acne for 30EE/DRSP have been performed, both with a non-inferiority design. Such trials differ methodologically from the more typical superiority design. A primary consideration in such studies is that the efficacy of the comparator is established or is used widely such that a placebo design would be considered unethical. In contrast to superiority trials, the null hypothesis in non-inferiority trials is that the treatments differ by a pre-established margin. The pre-stated margins of non-inferiority can be established as the smallest value representing a clinically relevant effect or a value smaller than the effect chosen to investigate superiority over placebo. With non-inferiority trials, type I error reflects incorrect acceptance of an inferior new treatment and type II error is incorrect rejection of a non-inferior treatment. Lastly, analysis of per-protocol groups rather than intent to treat as in superiority trials reduces the risk of type I error in non-inferiority trials. ${ }^{33}$ No active-comparator trials are available for 20EE/DRSP to date.

\section{Comparison to $35 \mu \mathrm{g}$ ethinyl estradiol/2 mg cyproterone acetate ${ }^{34}$}

The efficacy of 30EE/DRSP versus $35 \mu \mathrm{g}$ ethinyl estradiol $/ 2 \mathrm{mg}$ cyproterone acetate (EE/CPA) was evaluated in an active-comparator trial with non-inferiority design. This 9-cycle double-blind randomized controlled trial had the following inclusion criteria: women 16 to 35 years, mild-tomoderate facial acne, and a minimum of 8 papulo-pustules.
Standard exclusion criteria regarding contraindications for combined OC use and acne treatments applied.

The primary efficacy outcome was the change in total acne lesion count from baseline to end of study at the 9th cycle. The null hypothesis, that 30EE/DRSP was inferior to $\mathrm{EE} / \mathrm{CPA}$ relative to change in total acne lesion counts, was tested against the alternative hypothesis of non-inferiority with a margin of $25 \%$.

One hundred and twenty-eight women were recruited and randomized in a 2:1 ratio to receive 30EE/DRSP or EE/ CPA, respectively. Baseline demographics between groups were similar. The intent to treat (ITT) population comprised subjects who had taken trial medication at least once (total $\mathrm{N}=125 ; 30 \mathrm{EE} / \mathrm{DRSP}, 82$ and EE/CPA, 43). Of these, those who completed the study without major protocol violations comprised the per protocol (PP) population (total $\mathrm{N}=91$; 30EE/DRSP, 58, EE/CPA, 33).

In the PP population, mean total lesion count reductions were $46.8 \%$ ( $\mathrm{SD} \pm 42.2$ ) for $30 \mathrm{EE} / \mathrm{DRSP}$ and $40.4 \%$ (SD \pm 70.2 ) for EE/CPA. In both ITT and PP datasets 30EE/DRSP was found to be noninferior to EE/CPA after 9 cycles $(P=0.0006)$. Non-inflammatory lesion counts were reduced by $50 \%$ (from mean of 23 to 9 ) and $60 \%$ (from 21 to 6 ) in the 30EE/DRSP and EE/CPA groups respectively. Corresponding reduction in inflammatory lesion counts (papules and pustules) were $73.5 \%$ (from 16 to 4 ) and $75 \%$ (from 20 to 5) respectively.

One serious adverse event (shoulder-arm syndrome) was reported in the 30EE/DRSP group, but was considered unrelated to treatment. Both preparations were well tolerated and adverse events typical of low-dose combined oral contraceptive therapy were largely mild-to-moderate in severity 
Table 2 Pooled outcomes of $20 \mu \mathrm{g}$ EE/3 mg DRSP vs placebo trials in acne ${ }^{29,30}$

\begin{tabular}{lll}
\hline Outcome & \multicolumn{2}{l}{ Pooled results } \\
\cline { 2 - 3 } & Active & Placebo \\
\hline pooled IGA success & $18 \%$ & $6 \%$ \\
$\%$ reduction total lesions & $44 \%$ & $28 \%$ \\
\% reduction inflammatory lesions & $49 \%$ & $33 \%$ \\
\% reduction noninflammatory lesions & $40 \%$ & $22 \%$ \\
\hline
\end{tabular}

Note: data pooling weighted for number of subjects.

and similar in both groups. No clinically significant changes in heart rate, blood pressure, or laboratory parameters were noted in either group. Weight gain was observed in $2.4 \%$ of those on 30EE/DRSP and 9.3\% on EE/CPA. Breast pain was less frequently reported in the 30EE/DRSP group: $8.5 \%$ compared to $16.3 \%$ on EE/CPA.

This study showed that 30EE/DRSP was non-inferior to $\mathrm{EE} / \mathrm{CPA}$ over 9 cycles in total acne lesion count reduction, with a non-inferiority margin of $25 \%$. There are, however, methodological issues with this study. It is unclear how the non-inferiority margin of $25 \%$ was determined. Previous placebo-controlled trials of other combined OCs have found a significant difference in total lesion counts after 6 cycles of $15 \%$ to $20 \% .{ }^{35-38}$ Accordingly, a non-inferiority margin beyond this range would be considered excessive. Of additional concern is that no information is provided on the power of the study or the related determination of sample size. Furthermore, the clinically relevant measure of global assessment grading was not used in this study. Since clinicians typically use global assessments in clinical practice rather than lesion counts, the generalizability of these results is limited.

\section{Comparison to a triphasic preparation containing $35 \mu \mathrm{g}$ ethinyl estradiol and norgestimate 39}

The triphasic preparation $35 \mu \mathrm{g}$ EE/0.180, 0.215, $0.250 \mathrm{mg}$ norgestimate (EE/NGM; Pramino ${ }^{\circledR}$, Ortho Tri-Cyclen ${ }^{\circledR}$ ) has previously been demonstrated in 2 placebo-controlled, double-blind randomized trials to be effective in treatment of moderate facial acne. ${ }^{35,36}$

Designed in a similar manner to these studies, a comparator study of 30EE/DRSP with EE/NGM was randomized, prospective, and double-blinded with the same inclusion criteria. Specifically, these comprised healthy females aged 15 to 40 years with mild to moderate facial acne vulgaris and no contraindications to combined oral contraceptive use.
Subjects were required to have 6 to 100 non-inflammatory lesions, 10 to 50 papules and/or pustules, and less than 5 nodules. However, unlike the superiority design of the earlier placebo-controlled studies, this study was designed as a non-inferiority trial. Participants were randomized in a 1:1 ratio to 30EE/DRSP or EE/NGM for 6 treatment cycles. Efficacy outcomes included lesion counts and retrospective investigator assessments of therapeutic effect based on recall of baseline severity. A static global assessment was not performed.

This study tested the hypothesis that EE/DRSP was noninferior by a margin of $10 \%$ to $\mathrm{EE} / \mathrm{NGM}$ at improving the lesion counts and proportion of subjects with improvement based on investigator assessment. The calculated minimum sample size for the each of the primary variables based on power of $90 \%$ and a one-sided significance level of $2.5 \%$ was 338 for each treatment group.

A total of 1154 subjects were randomized to either 30EE/ DRSP $(n=568)$ or EE/NGM $(n=586)$ groups. Of these, 566 assigned to $30 \mathrm{EE} / \mathrm{DRSP}$ and 582 to EE/NGM comprised the ITT group while 486 in the 30EE/DRSP and 505 in the EE/NGM were included in the PP group. For the purpose of non-inferiority analysis, the PP group was subjected to further evaluation while the ITT group was used for analysis of superiority.

In the PP group, mean inflammatory lesion counts were reduced by $76 \%(\mathrm{SD} \pm 26)$ and $72 \%(\mathrm{SD} \pm 28)$ in the 30EE/DRSP and EE/NGM groups respectively after 6 cycles. Total lesion counts were reduced by $68 \%(\mathrm{SD} \pm 24)$ in the EE/DRSP group and $65 \%(\mathrm{SD} \pm 26)$ in the EE/NGM group after 6 cycles. These differences in favor of 30EE/DRSP (3\%) demonstrated non-inferiority to EE/NGM at the $10 \%$ level for total and inflammatory lesion counts. On analysis with the ITT population, these differences were shown to be significant compared to EE/NGM $(95 \% \mathrm{CI},-6.5$ to -0.1 ; $P=0.020$ ). Investigators assessment of acne improvement based on comparison to baseline demonstrated that $97 \%$ on $30 \mathrm{EE} / \mathrm{DRSP}$ compared to $94 \%$ on EE/NGM showed improvement in facial acne. This difference of $3 \%$ was within the non-inferiority margin of $10 \%$ and significantly favored 30EE/DRSP (95\% CI, 0.8 to $6.3 ; P=0.006$ ).

Adverse events related to treatment were mostly of mild or moderate intensity and did not cause discontinuation of treatment. Those events reported by over $2 \%$ of subjects in the 30EE/DRSP and EE/NGM groups were typical of other combined oral contraceptives and included nausea $(4.8 \%$ vs $5.5 \%$ ), headache (3.9\% vs $3.6 \%$ ), breast pain ( $2.8 \%$ vs $2.9 \%)$, and abdominal pain ( $1.4 \%$ vs $2.2 \%)$. No significant changes 
were reported in safety laboratory parameters, physical and gynecological examination, or vital signs. No serious treatment-related adverse events were reported in the 30EE/DRSP group, while there were 2 serious adverse events in the EE/NGM group considered to be possibly related to treatment (migraine and fibrocystic breast). Of the 2 pregnancies in this study, 1 subject in the 30EE/DRSP group became pregnant during the trial after discontinuing study medication, while another subject in the EE/NGM group became pregnant due to poor treatment compliance.

This large study was adequately powered to demonstrate noninferiority of 30EE/DRSP compared to EE/NGM with respect to lesion counts and retrospective investigator assessment with a margin of $10 \%$. While $30 \mathrm{EE} / \mathrm{DRSP}$ was also shown to be statistically superior to EE/NGM in reduction of total lesions $(3.3 \%)$ and retrospective investigator assessments (3.6\%), the magnitude of these differences is not likely to be clinically relevant. A shortcoming of this study is the use of an investigator assessment referencing baseline severity. Such an outcome measure is flawed by recall bias and the potential use of lesion counts to determine clinical improvement. The latter would relegate this pivotal clinical measure to that of a dependent variable rather than maintaining its role as an independent efficacy outcome.

\section{Trials on safety}

The evaluation of thromboembolic risk for combined OCs requires many more subjects than typically available in smaller efficacy studies in acne. For this class of agents, the potential for hyperkalemia due to the antimineralocorticoid activity of DRSP and potential for arrhythmias has also been of concern. Accordingly, the safety of DRSP-containing OCs has been further scrutinized in mandated large post-marketing surveillance studies.

Two large studies have been conducted to evaluate the risk of vascular thromboembolic disease for 30EE/DRSP compared to other OCs. ${ }^{40,41}$ In a United States population using a claims database maintained by United Health Care, the incidence of venous thromboembolism (VTE) was evaluated for 22,429 women initiating 30EE/DRSP and a matched group of 44,858 initiating other OCs. ${ }^{40}$ The average duration of follow-up was 7.8 and 7.5 months, respectively. VTE was confirmed in 18 cases of 30EE/DRSP users and in 39 using other OCs. This translated to VTE incidence rates of 1.3/1000 woman years for those on 30EE/DRSP and 1.4/1000 woman years for those on other OCs. The VTE subgroups of deep vein thrombosis and pulmonary embolism were equal in frequency between these 2 groups.
An European prospective trial of initiators of DRSP compared to levonorgestrel and other progestin-containing OCs, observed that the incidence of both venous and arterial thromboembolic events did not differ significantly between groups. This study comprised 58,674 women followed for 142,475 women-years and demonstrated that VTE hazard ratios for DRSP-containing versus LNGcontaining OCs and other OCs were 1.0 and 0.7 (upper 95\% CI, 1.8 and 1.3, respectively). Corresponding values for arterial thromboembolism were 0.3 and 0.3 (upper $95 \%$ CI, 1.2 and 1.5, respectively). ${ }^{41}$ Arrhythmias suggestive of hyperkalemia were rare in both groups with an incidence was $0.28 / 1000$ woman years for those on $30 \mathrm{EE} / \mathrm{DRSP}$ compared to $0.26 / 1000$ woman years for those in the comparator group. Thus, these studies demonstrated no increased risk of vascular thromboembolic disease or arrhythmias for DRSPcontaining OCs.

\section{Summary}

DRSP is currently only available with ethinyl estradiol as a combined $\mathrm{OC}$ in one of 2 preparations varying in dose of $\mathrm{EE}$ and in duration of hormone/hormone-free intervals: $30 \mu \mathrm{g}$ $\mathrm{EE} / 3 \mathrm{mg}$ DRSP in a $21 / 7$ regimen and a $20 \mu \mathrm{g} \mathrm{EE} / 3 \mathrm{mg}$ DRSP in a 24/4 regimen. In treatment of acne, these agents address the androgenic factors operative in acne pathogenesis while also providing reliable contraception. The unique advantage of DRSP as the progestin in such preparations lies in its antimineralocorticoid and antiandrogenic effects. They are particularly well tolerated, being devoid of the fluid retentional effects of OCs containing other progestins. Additionally, their relative safety has been demonstrated in large epidemiological surveillance studies.

The theoretical benefits of the antiandrogenic potential of DRSP-containing OCs in acne have been recently demonstrated in both placebo-controlled superiority and activecomparator non-inferiority trials. Additionally, the superiority of 30EE/DRSP over EE/NGM (another OC approved for use in acne) in acne has been demonstrated statistically. While the difference in outcomes favoring 30EE/DRSP was of marginal clinical relevance, this is one of the only available trials demonstrating the superiority of one OC preparation over another in the treatment of acne. Future comparative evaluations of OC preparations would assist clinicians in selection of acne treatment within this class of agents.

For management of mild to moderate acne in women, OCs will likely be used with topical medications such as retinoids and antibacterial agents in standard clinical practice. Such combination therapy should lead to more rapid onset of effect 
and greater magnitude of improvement given the synergistic mechanisms of these different treatments. Future studies should evaluate the outcomes of such combination therapy as it is more reflective of clinical practice. Additionally, investigations regarding the antiandrogenic dose equivalency of DRSP to spironolactone are especially pertinent in view of the widespread use of the latter in treatment of acne. In severe acne, the use of DRSP-containing OCs may reduce the risk of additional adverse events associated with other combined OCs in women requiring contraception when prescribed oral isotretinoin. In conclusion, across the spectrum of clinical acne severity, the availability of DRSP-containing OCs with demonstrated efficacy in acne along with their excellent tolerability and safety profiles suggest that they should be considered to be the current standards of hormonal therapy for women with acne.

\section{Abbreviations}

OC, oral contraceptive; EE, ethinyl estradiol; DRSP, drosperinone; DHT, dihydrotestosterone; SHBG, sex hormone binding globulin; CDER, Center of Drug Evaluation and Research; IGA, investigator global assessments; 20EE/DRSP, $20 \mu \mathrm{g}$ EE/3 mg DRSP; 30EE/DRSP, $30 \mu \mathrm{g}$ EE/3 mg DRSP; $\mathrm{EE} / \mathrm{CPA}, 35 \mu \mathrm{g}$ ethinyl estradiol/2 mg cyproterone acetate; EE/NGM, triphasic preparation $35 \mu \mathrm{g}$ EE/0.180, 0.215, $0.250 \mathrm{mg}$ norgestimate; VTE, venous thromboembolism.

\section{Disclosures}

DrTan has served as a consultant and speaker for Berlex Canada, and as a speaker for Bayer Healthcare Pharmaceuticals.

\section{References}

1. Kilkenny M, Merlin K, Plunkett A, Marks R. The prevalence of common skin conditions in Australian school students: 3. Acne vulgaris. Br J Dermatol. 1998;139(5):840-845.

2. Lello J, Pearl A, Arroll B, Yallop J, Birchall NM. Prevalence of acne vulgaris in Auckland senior high school students. NZ Med J. 1995;108(1004):287-289.

3. Goulden V, Stables GI, Cunliffe WJ. Prevalence of facial acne in adults. J Am Acad Dermatol. 1999;41(4):577-580.

4. Collier CN, Harper JC, Cantrell WC, Wang W, Foster KW, Elewski BE. The prevalence of acne in adults 20 years and older. J Am Acad Dermatol. 2008;58(1):56-59.

5. Shaw JC, White LE. Persistent acne in adult women. Arch Dermatol. 2001;137(9):1252-1253.

6. Kellett SC, Gawkrodger DJ. The psychological and emotional impact of acne and the effect of treatment with isotretinoin. Br J Dermatol. 1999; 140(2):273-282.

7. Gupta MA, Gupta AK. Depression and suicidal ideation in dermatology patients with acne, alopecia areata, atopic dermatitis and psoriasis. Br J Dermatol. 1998;139(5):846-850.

8. Krowchuk DP, Stancin T, Keskinen R, Walker R, Bass J, Anglin TM. The psychosocial effects of acne on adolescents. Pediatr Dermatol. 1991;8(4):332-338.
9. Tan JKL, Li Y, Fung K, et al. Divergence of demographic factors associated with clinical severity compared to QoL impact in acne. J Cutan Med Surg. 2008;12(5):235-242.

10. Tan J. New Developments in Hormonal Therapy for Acne. Skin Therapy Lett 2007;12(7):1-3.

11. Carmina E, Lobo R. A comparison of the relative efficacy of antiandrogens for the treatment of acne in hyperandrogenic women. Clin Endocrinol (Oxf). 2002;57(2):231-234.

12. Shaw J, White LE. Long-term safety of spironolactone in acne: results of an 8 year followup study. J Cutan Med Surg. 2002;6(6):541-545.

13. Gollnick H, Cunliffe W, Berson D, et al. Management of acne: a report from a global alliance to improve outcomes in acne. $\mathrm{J} \mathrm{Am} \mathrm{Acad}$ Dermatol. 2003;43(1 Supp):S1-S37.

14. O'Connell K, Westhoff C. Pharmacology of Hormonal Contraceptives and Acne. Cutis. 2008;81(1 Suppl):8-12.

15. Thiboutot D. Regulation of Human Sebaceous Glands. J Invest Dermatol. 2004;123(1):1-12.

16. Muhn P, Krattenmacher R, Beier S, Elger W, Schillinger E. Drospirenone: a novel progestogen with antimineralocorticoid and antiandrogenic activity. Pharmacological characterization in animal models. Contraception. 1995;51(2):99-110.

17. Oelkers W, Berger V, Bolik A, et al. Dihydrospirorenone, a new progestogen with antimineralocorticoid activity: Effects on ovulation, electrolyte excretion, and the renin-aldosterone system in normal women. J Clin Endocrinol Metab. 1991;73(4):837-842.

18. Krattenmacher R, Fromm M. Mineralocorticoid and antimineralocorticoid effects of various progestogens on electrogenic $\mathrm{Na}^{+}$transport in the rat distal colon in vitro. [abstract] 36th Symposion of the "Deutsche Gesellschaft fur Endokrinologie.” Acta Endocrinol. 1992;124:88.

19. Fuhrmann U, Krattenmacher R, Slater EP, Fritzemeier KH. The novel progestin drospirenone and its natural counterpart progesterone: biochemical profile and antiandrogenic potential. Contraception. 1996;54(4):243-251.

20. Pollow K, Juchem M, Elger W, Jacobi N, Hoffmann G, Mobus V. Dihydrospirorenone (ZK 30595): A novel synthetic progestagencharacterization of binding to different receptor proteins. Contraception. 1992;46(6):561-574.

21. Elger W, Beier S, Pollow K, Garfield R, Shi SQ, Hillisch A. Conception and pharmacodynamic profile of drospirenone. Steroids. 2003; 68(10-13):891-905.

22. Losert W, Casals-Stenzel J, Buse M. Progestogens with antimineralocorticoid activity. Arzneimittelforschung. 1985;35(2):459-471.

23. YAZ drosperinone and ethinyl estradiol tablets. Prescribing information. Bayer Health Care Pharmaceuticals Inc. Wayne, New Jersey. 2007. www.yazus.com/hcp/pdf/Prescribing_Information_Key_Points.pdf. Accessed Sept 2009.

24. Schindler AE, Campagnoli C, Druckmann R, et al. Classification and pharmacology of progestins. Maturitas.2003;46(Supp 1):S7-S16.

25. Lehmann HL, Robinson KA, Andrews JS, Holloway V, Goddman SN. Acne therapy: A methodological review. J Am Acad Dermatol. 2002; 47(2 Pt 1):231-240.

26. Dermatologic and Opthalmic Drugs Advisory Committee. Executive summary. 2002 Nov 4-5[about 1 p.]. www.fda.gov/ohrms/dockets/ac/02/ briefing/3904B1_02_Executive\%20Summary.htm. Accessed Sept. 2009.

27. Tan JK. Current measures for the evaluation of acne severity. Exp Rev Derm. 2008;3(5):595-603.

28. Willis SA, Kuehl TJ, Spikerman AM, Sulak PJ. Greater inhibition of the pituitary-ovarian axis in oral contraceptive regimens with a shortened hormone-free interval. Contraception. 2006;74(2):100-103.

29. Maloney JM, Dietze P Jr, Watson D, et al. Treatment of acne using a 3-milligram drospirenone/20-microgram ethinyl estradiol oral contraceptive administered in a 24/4 regimen: a randomized controlled trial. Obstet Gynecol. 2008;112(4):773-781.

30. Koltun W, Lucky AW, Thiboutot D, et al. Efficacy and safety of $3 \mathrm{mg}$ drospirenone $/ 20 \mathrm{mcg}$ ethinylestradiol oral contraceptive administered in 24/4 regimen in the treatment of acne vulgaris: a randomized, doubleblind, placebo-controlled trial. Contraception. 2008;77(4):249-256. 
31. Maloney JM, Dietze P Jr, Watson D, et al. A randomized controlled trial of a low-dose combined oral contraceptive containing $3 \mathrm{mg}$ drospirenone plus $20 \mu \mathrm{g}$ ethinylestradiol in the treatment of acne vulgaris: lesion counts, investigator ratings and subject self-assessment. J Drugs Dermatol. 2009;8(9):837-844.

32. Lucky AW, Koltun W, Thiboutot D, et al. A combined oral contraceptive containing $3 \mathrm{mg}$ drospirenone/20 ug ethinyl estradiol in the treatment of acne vulgaris: a randomized, double-blind placebo-controlled study evaluating lesion counts and participant self assessment. Cutis. 2008;82(2):143-150

33. Piaggio G, Elbourne DR, Altman DG, Pocock SJ, Evans SJW. Reporting of noninferiority and equivalence randomised trials. JAMA. 2006:295(10):1152-1160.

34. Van Vloten WA, van Haselen CW, van Zuuren EJ, Gerlinger C, Heithecker $\mathrm{R}$. The effect of 2 combined oral contraceptives containing either drosperinone or cyproterone acetate on acne and seborrhea. Cutis. 2002;69(4 Suppl):2-15.

35. Lucky AW, Henderson TA, Olson WH, Robisch DM, Lebwohl M, Swinyer LJ. Effectiveness of norgestimate and ethinyl estradiol in treating moderate acne vulgaris. JAm Acad Dermatol. 1997;37(5 Pt 1): 746-754

36. Redmond GP, Olson WH, Lippman JS, Kafrissen ME, Jones TM, Jorizzo JL. Norgestimate and ethinyl estradiol in the treatment of acne vulgaris: a randomized, placebo-controlled trial. Obstet Gynecol. 1997;89(4):615-622.
37. Leyden J, Shalita A, Hordinsky Mswinyer L, Stanczyk FZ, Weber ME Efficacy of a low-dose oral contraceptive containing $20 \mu \mathrm{g}$ of ethinyl estradiol and $100 \mu \mathrm{g}$ of levonorgestrel for the treatment of moderate acne: A randomized, placebo-controlled trial. J Am Acad Dermatol. 2002;47(3):399-409.

38. Thiboutot D, Archer DF, Lemay A, Washenik K, Roberts J, Harrison DD. A randomized, controlled trial of a low-dose contraceptive containing $20 \mu \mathrm{g}$ of ethinyl estradiol and $100 \mu \mathrm{g}$ of levonorgestrel for acne treatment. Fertil Steril. 2001;76(3):461-468.

39. Thorneycroft IH, Gollnick H, Schellschmidt I. Superiority of a combined contraceptive containing drosperinone to a triphasic preparation containing norgestimate in acne treatment. Cutis. 2004;74(2):123-130.

40. Seeger JD, Loughlin J, Eng PM, Clifford CR, Cutone J, Walker AM. Risk of thromboembolism in women taking ethinylestradiol/drospirenone and other oral contraceptives. Obstet Gynecol. 2007;110(3):587-593.

41. Dinger JC, Heinemann LA, Kühl-Habich D. The safety of a drospirenonecontaining oral contraceptive: final results from the European Active Surveillance study on oral contraceptives based on 142,475 women-years of observation. Contraception. 2007;75(5):344-354.
International Journal of Women's Health

\section{Publish your work in this journal}

The International Journal of Women's Health is an international, peerreviewed open-access journal publishing original research, reports, reviews and commentaries on all aspects of women's healthcare including gynaecology, obstetrics, and breast cancer. Subject areas include: Chronic conditions (migraine headaches, arthritis, osteoporosis);

\section{Dovepress}

Endocrine and autoimmune syndromes; Sexual and reproductive health; Psychological and psychosocial conditions. The manuscript management system is completely online and includes a very quick and fair peer-review system. Visit http://www.dovepress.com/ testimonials.php to read real quotes from published authors. 